\title{
Errata
}

Phys. Chem. Minerals (1993) 20:228-241

\section{Energy and Long-Range Disorder in Simple Spinels}

\section{Antonio Della Giusta ${ }^{1}$ and Giulio Ottonello ${ }^{2}$}

${ }^{1}$ Dipartimento di Mineralogia e Petrologia, Università di Padova, Corso Garibaldi 37, I-35100 Padova, Italy

${ }^{2}$ Dipartimento di Scienze della Terra, Università di Cagliari, Via Trentino 51, I-07100 Cagliari, Italy

p. 229: Equation (14) should read:

$U_{v}=f\left(\frac{T}{\theta_{c}}\right)$

p. 237 right column, lines 16 and 20 from top:

Replace $\delta \mathrm{U}$ by $\delta \mathrm{u}$.

p. 238: Equation (27) should read:

$C p=C p_{c}+C p_{v}$

Equation (28) should read:

$S c_{(T)}-S c_{\left(T_{r}\right)}=\int_{T_{r}}^{T}\left(K 1_{c}+K 2_{c} T+K 3_{c} T^{-2}\right) \frac{\mathrm{d} T}{T}$

Equation (30) should read:

$\Delta G_{C\left(T-T_{r}\right)}=\int_{T_{r}}^{T} C p_{c} \mathrm{~d} T-T \int_{T_{r}}^{T} C p_{c} \frac{\mathrm{d} T}{T}$

p. 240: Equation (A2) should read:

$\Delta_{\mathrm{o}}=\frac{e \mu_{d} a^{4}}{\bar{D}_{\lambda, j}^{5}}=\frac{e z a^{4}}{\bar{D}_{i, j}^{5}}$ 